\title{
M-Polynomials and Associated Topological Indices of Sodalite Materials
}

\author{
Ghazanfar Abbas, ${ }^{1}$ Muhammad Ibrahim $\left(\mathbb{D},{ }^{1}\right.$ Ali Ahmad $(D), 2$ Muhammad Azeem (D), \\ and Kashif Elahi $\mathbb{D}^{4}$ \\ ${ }^{1}$ Centre for Advanced Studies in Pure and Applied Mathematics, Bahauddin Zakariya University, Multan, Pakistan \\ ${ }^{2}$ College of Computer Science \& Information Technology, Jazan University, Jazan, Saudi Arabia \\ ${ }^{3}$ Department of Aerospace Engineering, Faculty of Engineering, Universiti Putra Malaysia, Seri Kembanga, Malaysia \\ ${ }^{4}$ Deanship of E-Learning and Information Technology, Jazan University, Jazan, Saudi Arabia
}

Correspondence should be addressed to Muhammad Ibrahim; mibtufail@gmail.com

Received 9 August 2021; Accepted 6 September 2021; Published 1 October 2021

Academic Editor: Amin Jajarmi

Copyright (c) 2021 Ghazanfar Abbas et al. This is an open access article distributed under the Creative Commons Attribution License, which permits unrestricted use, distribution, and reproduction in any medium, provided the original work is properly cited.

\begin{abstract}
Natural zeolites are commonly described as macromolecular sieves. Zeolite networks are very trendy chemical networks due to their low-cost implementation. Sodalite network is one of the most studied types of zeolite networks. It helps in the removal of greenhouse gases. To study this rich network, we use an authentic mathematical tool known as M-polynomials of the topological index and show some physical and chemical properties in numerical form, and to understand the structure deeply, we compare different legitimate M-polynomials of topological indices, concluding in the form of graphical comparisons.
\end{abstract}

\section{Introduction}

Macromolecular sieves, commonly known as natural zeolites, are becoming more investigated and researched [1-3]. The custom-made molecular capturing of zeolite makes it significant and very applicable. Due to their lower costs, natural zeolites are considered as useful in bulk mineral applications [4]. According to the complexity and varying sizes, zeolites are characterized into different groups. For example, tschernichite, stilbite, mordenite, chabazite, faujasite, sodalite and Linde type A are most useful in terms of commercial applications, molecular sieves, or natural zeolites [5]. Among all the zeolite structures stated above, synthetic compounds and mineral's crystal structure with the frameworks of sodalite structure are the enormously studied compounds [6,7]. Some physical and chemical properties of sodalite and zeolite type structures in terms of topological indices can be found in [8-10].

The sodalites with the highest thermodynamic stability are considered as one of the top ranked structures among all the zeolites $[11,12]$. The sodalites are also considerable due to their crystallographic standpoints [13]. The sodalite structure's unit cell is built up by two cages. The six and four membered rings represent the basic topology of each cage. These rings are also shared by two parallel cages. To trap the molecules, in the sodalite, cavities are built up by tailor-made composition. Without damaging the crystal structures, tap molecules like water and $\mathrm{CO}_{2}$ have ability of desorbing and adsorbing molecules. As a result, for the removal of greenhouse gases and water, zeolites can be used [14]. For further studies related to zeolites, sodalite, and its useful applications, we refer the readers to $[15,16]$.

As we know that the study of M-polynomial is a very useful combination of numerical descriptors and algebraic theory, for the study of chemical networks, one can find an intense study on this topic; very particular and selected articles are cited here. Nanotube-related networks or structures were studied in [17-19], M-polynomials for different generalized families of graphs were discussed in [20-22], convex polytopes were studied in [23], chemical 
benzenoid structures were discussed in [24], a fine relation of M-polynomial with the probabilistic theory was discussed in [25], the study of this topic on metal organic structure was detailed in $[26,27]$, and some computer-related networks in terms of M-polynomials can be found in [28, 29]; there are many types of polynomials, and one of the varieties can be found in [30].

Topological index is a function in numerical form and describes the biological, chemical, and physical properties of given molecular graph in domain by a systematic way. Few very interesting and highly recommended topological indices are given in the following definitions along with their M-polynomials. The researchers in [31] studied polyhex and prism structures in terms of some M-polynomials. The titania nanotubes were studied in terms of some M-polynomials in [32]. The structure of nanostar dendrimers and polyhex nanotubes was studied in [33]. For few interesting studies of chemical structures and their topological properties and different shapes, we refer the readers to $[34,35]$. There are many ways and types of computing topological indices for different structures, for example, Shabbir et al. [36] computed the edge-based interpolation with the degree of vertices and topological indices, Nadeem et al. and Hong et al. [37, 38] computed the topological indices for the metal organic structure; line graph of some families of graphs and their topological indices are resulted in $[39,40]$, some ev- and ve-degree-based descriptors are studied in [41, 42], and the topological descriptors for some computer-related structures are discussed in $[43,44]$. Some neural networks and their topological descriptors were discussed in $[45,46]$.

To compute our major results, we need to define some basics; for example, let $\chi$ be a graph with vertex set $\chi$ and edge set $E(\chi)$; their cardinalities are represented by $n_{\chi}$ and $m_{\chi}$, respectively. Degree of a vertex (say $\lambda_{\mathfrak{a}}$ ) is the cardinality of incident edges with a vertex. There is another way to define edge set, which is called edge partition, usually written as $\left(\xi_{\mathfrak{a}}, \xi_{\mathfrak{b}}\right)$, where $\xi_{\mathfrak{a}}, \xi_{\mathfrak{b}}$ are the degrees of vertices $\mathfrak{a}$ and $\mathfrak{b}$, respectively, and $\mathfrak{a}, \mathfrak{b}$ create an edge say $\mathfrak{a} \mathfrak{b}$.

Following are some useful definitions of M-polynomials which are helpful to find our main results and create formulas for M-polynomials of different topological indices.

Definition 1. In 1988, Hosoya polynomial was introduced in [47]; with a relevance to Hosoya polynomial, in 2015, modified polynomial or usually named as M-polynomial was given in [48]. This particular type of polynomial is closely related to degree-based topological indices. Using a particular type of format, one can get the topological indices from M-polynomials of a graph. This M-polynomial can be defined as

$$
M(\chi ; x, y)=\sum_{i \leq j} m_{i, j}(\chi) x^{i} y^{j},
$$

where $m_{i, j}(\chi)$ is the number of edges of graph $\chi$ such that $i \leq j$.
Definition 2. The first and second Zagreb indices were introduced in 1972 by Gutman [49, 50], and their M-polynomials are defined as [48]

$$
\begin{aligned}
M_{1}(\chi) & =\sum_{\mathfrak{a} \mathfrak{b} \in E(\chi)}\left(\xi_{\mathfrak{a}}+\xi_{\mathfrak{b}}\right), \\
M_{2}(\chi) & =\sum_{\mathfrak{a} \mathfrak{b} \in E(\chi)}\left(\xi_{\mathfrak{a}} \times \xi_{\mathfrak{b}}\right), \\
P_{M_{1}}(\chi) & =\left(D_{x}+D_{y}\right)(M(\chi ; x, y)), \\
P_{M_{2}}(\chi) & =\left(D_{x} D_{y}\right)(M(\chi ; x, y)) .
\end{aligned}
$$

Definition 3. The second modified Zagreb index was given in [51], and its M-polynomial is defined as [48]

$$
\begin{aligned}
{ }^{m} M_{2} M_{2}(\chi) & =\sum_{\mathfrak{a} \mathfrak{b} \in E(\chi)} \frac{1}{\xi_{\mathfrak{a}} \times \xi_{\mathfrak{b}}}, \\
P^{m} M_{2} & (\chi)=\left(S_{x} S_{y}\right)(M(\chi ; x, y)) .
\end{aligned}
$$

Definition 4. In 1988, Bollobas and Erdos and Amic et al. $[52,53]$ proposed the general Randić index independently. Following are general Randić index, general inverse Randić index, and their M-polynomials [48]:

$$
\begin{aligned}
R_{\alpha}(\chi) & =\sum_{u v \in E(\chi)}\left(\xi_{\mathfrak{a}} \times \xi_{\mathfrak{b}}\right)^{\alpha}, \\
P_{R_{\alpha}}(\chi) & =\left(D_{x}^{\alpha} D_{y}^{\alpha}\right)(M(\chi ; x, y)), \\
\operatorname{IR}_{\alpha}(\chi) & =\sum_{\mathfrak{a} \mathfrak{b} \in E(\chi)}\left(\frac{1}{\xi_{\mathfrak{a}} \times \xi_{\mathfrak{b}}}\right)^{\alpha}, \\
P_{\mathrm{IR}_{\alpha}}(\chi) & =\left(S_{x}^{\alpha} S_{y}^{\alpha}\right)(M(\chi ; x, y)),
\end{aligned}
$$

where

$$
\begin{aligned}
D_{x}(f(x, y)) & =x \frac{\partial f}{\partial x}, \\
D_{y}(f(x, y)) & =x \frac{\partial f}{\partial y}, \\
S_{x}(f(x, y)) & =\int_{0}^{x} \frac{f(z, y)}{z} \mathrm{~d} z, \\
S_{y}(f(x, y)) & =\int_{0}^{y} \frac{f(x, z)}{z} \mathrm{~d} z .
\end{aligned}
$$

In this research work, we studied sodalite's three-dimensional structure which is an important compound of zeolite network, in terms of different M-polynomials of topological indices defined above. Moreover, we explain the structure more precisely by taking different numerical examples and doing comparative study between computed M-polynomials, and at the end, we conclude the study in the form of graphical representations of derived numerical examples. 


\section{Results of M-Polynomials on Sodalite Network}

The graph shown in Figure 1 is the three-dimensional sodalite network $S_{p, q, r}$. It contains $4(p q+p r+q r)+12 p q r$ total number of vertices and $4(p q+p r+q r)+524 p q r$ total number of edges. As shown in Figure 1, it contains two types of vertices and three types of edges, which are useful for our main results. Figure 1 is studied in [14] in detail to explore some useful structures related to sodalite and zeolites. Now, the first result is the main and general M-polynomial of sodalite network $S_{p, q, r}$.

Theorem 1. Let $S_{p, q, r}$ be a sodalite material, with $p, q, r \geq 1$, whose structure can be seen in Figure 1. Then, its M-polynomial is

$$
\begin{aligned}
M\left(S_{p, q, r}, x, y\right)= & 24 p q r x^{4} y^{4}+4(p q+p r+q r) \\
& \left(2 x^{3} y^{3}+2 x^{3} y^{4}-3 x^{4} y^{4}\right) \\
& +4(p+q+r)\left(x^{3} y^{3}-2 x^{3} y^{4}+x^{4} y^{4}\right)
\end{aligned}
$$

Proof. From Figure 1, which is the construction of sodalite materials, we can observe that there are two vertex set partitions:

$$
\begin{aligned}
& \bigvee_{3}=\left\{\theta \in V\left(S_{p, q, r}\right): d_{\theta}=3\right\} \\
& \bigvee_{4}=\left\{\theta \in V\left(S_{p, q, r}\right): d_{\theta}=4\right\}
\end{aligned}
$$

with $\left|\vee_{3}\right|=8(p q+p r+q r)$ and $\left|\vee_{4}\right|=12 p q r-4(p q+$ $p r+q r)$. Also, there are three edge partitions based on degree of end vertices of each edge that is defined as

$$
\begin{aligned}
& \Xi_{3,3}=\left\{\theta \lambda \in E\left(S_{p, q, r}\right): d_{\theta}=3, d_{\lambda}=3\right\}, \\
& \Xi_{3,4}=\left\{\theta \lambda \in E\left(S_{p, q, r}\right): d_{\theta}=3, d_{\lambda}=4\right\}, \\
& \Xi_{4,4}=\left\{\theta \lambda \in E\left(S_{p, q, r}\right): d_{\theta}=4, d_{\lambda}=4\right\} .
\end{aligned}
$$

The cardinality of these edge partitions is $m_{3,3}=\left|\Xi_{3,3}\right|=$ $8(p q+p r+q r)+4(p+q+r), \quad m_{3,4}=\left|\Xi_{3,4}\right|=8(p q+p r+$ $q r)-8(p+q+r)$, and $m_{4,4}=\left|\Xi_{4,4}\right|=24 p q r-12(p q+p r+$ $q r)+4(p+q+r)$. Then, from Definition 1, the M-polynomial of $S_{p, q, r}$ is

$$
\begin{aligned}
M\left(S_{p, q, r} ; x, y\right)= & \sum_{i \leq j} m_{i, j}\left(S_{p, q, r}\right) x^{i} y^{j} \\
= & m_{3,3}\left(S_{p, q, r}\right) x^{3} y^{3}+m_{3,4}\left(S_{p, q, r}\right) x^{3} y^{4} \\
& +m_{4,4}\left(S_{p, q, r}\right) x^{4} y^{4} \\
= & 24 p q r x^{4} y^{4}+4(p q+p r+q r) \\
& \left(2 x^{3} y^{3}+2 x^{3} y^{4}-3 x^{4} y^{4}\right) \\
& +4(p+q+r)\left(x^{3} y^{3}-2 x^{3} y^{4}+x^{4} y^{4}\right)
\end{aligned}
$$

Lemma 1. Let $S_{4,4,4}$ be a sodalite material, with $p, q, r \geq 1$. Then, the differential operator is

$$
\begin{aligned}
D_{x}= & 96 p q r x^{4} y^{4}+24(p q+p r+q r) \\
& \cdot\left(x^{3} y^{3}+x^{3} y^{4}-2 x^{4} y^{4}\right) \\
& +4(p+q+r)\left(3 x^{3} y^{3}-6 x^{3} y^{4}+4 x^{4} y^{4}\right), \\
D_{y}= & 96 p q r x^{4} y^{4}+8(p q+p r+q r) \\
& \cdot\left(3 x^{3} y^{3}+4 x^{3} y^{4}-6 x^{4} y^{4}\right) \\
& +4(p+q+r)\left(3 x^{3} y^{3}-8 x^{3} y^{4}+4 x^{4} y^{4}\right) .
\end{aligned}
$$

Proof. Differentiating equation (8) with respect to $x$ and multiplying the result with $x$, we get $D_{x}$. Similarly, differentiating equation (8) with respect to $y$ and multiplying the result with $y$, we get $D_{y}$.

Lemma 2. Let $S_{p, q, r}$ be a sodalite material, with $p, q, r \geq$. Then, the integral operator is

$$
\begin{aligned}
S_{x}= & 6 p q r x^{4} y^{4}+4(p q+p r+q r) \\
& \cdot\left(\frac{2}{3} x^{3} y^{3}+\frac{2}{3} x^{3} y^{4}-\frac{3}{4} x^{4} y^{4}\right) \\
& +4(p+q+r)\left(\frac{1}{3} x^{3} y^{3}-\frac{2}{3} x^{3} y^{4}+\frac{1}{4} x^{4} y^{4}\right), \\
S_{y}= & 6 p q r x^{4} y^{4}+4(p q+p r+q r) \\
& \cdot\left(\frac{2}{3} x^{3} y^{3}+\frac{1}{2} x^{3} y^{4}-\frac{3}{4} x^{4} y^{4}\right) \\
& +4(p+q+r)\left(\frac{1}{3} x^{3} y^{3}-\frac{1}{2} x^{3} y^{4}+\frac{1}{4} x^{4} y^{4}\right) .
\end{aligned}
$$

Proof. As we know from equation (6), $S_{x}=\int_{0}^{x}(M$ $\left.\left(S_{p, q, r} ; t, y\right) / t\right) \mathrm{d} t$ and using the general M-polynomial for the $S_{p, q, r}$ from equation (8) in it, after simplification, we obtain $S_{x}$. Similarly, from equation $(7), S_{y}=\int_{0}^{y}\left(M\left(S_{p, q, r} ; x, t\right) / t\right) \mathrm{d} t$ and using the general M-polynomial for the $S_{p, q, r}$ from equation (8) in it, after simplification, we obtain $S_{y}$.

Now, we will use these differential and integral operators from Lemmas 1 and 2 to obtain our main results. In the following theorem, we determined the M-polynomial of first and second Zagreb indices.

Theorem 2. Let $S_{p, q, r}$ be a sodalite material, with $p, q, r \geq 1$, and $P_{M_{1}}$ be the M-polynomial of first Zagreb index. Then, $P_{M_{1}}\left(S_{p, q, r}\right)$ is 


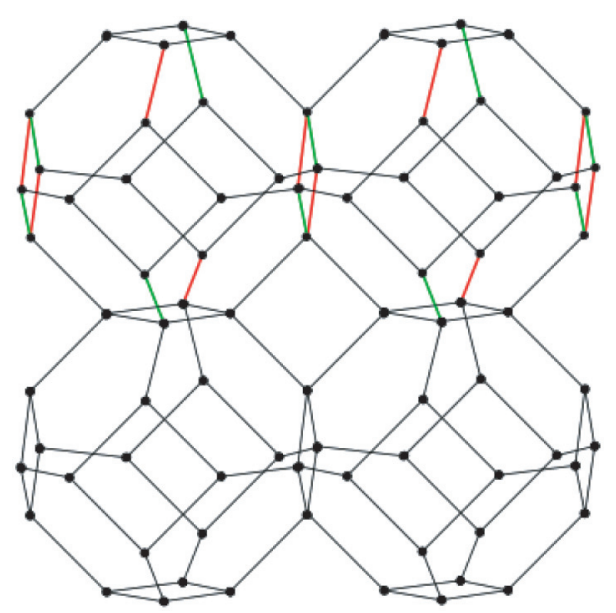

Figure 1: Sodalite network.

$$
\begin{aligned}
P_{M_{1}}\left(S_{p, q, r}\right)= & 192 p q r x^{4} y^{4}+8(p q+p r+q r)\left(6 x^{3} y^{3}+7 x^{3} y^{4}-12 x^{4} y^{4}\right) \\
& +8(p+q+r)\left(3 x^{3} y^{3}-7 x^{3} y^{4}+4 x^{4} y^{4}\right), \\
P_{M_{2}}\left(S_{p, q, r}\right)= & 384 p q r x^{4} y^{4}+24(p q+p r+q r)\left(3 x^{3} y^{3}+4 x^{3} y^{4}-8 x^{4} y^{4}\right) \\
& +4(p+q+r)\left(9 x^{3} y^{3}-24 x^{3} y^{4}+16 x^{4} y^{4}\right) .
\end{aligned}
$$

Proof. According to Definition 2, the formula for the M-polynomial of first Zagreb index for $S_{p, q, r}$ is defined as $P_{M_{1}}\left(S_{p, q, r}\right)=\left(D_{x}+D_{y}\right)\left(M\left(S_{p, q, r} ; x, y\right)\right)$, by using the differential operators that are defined in Lemma 1 for $S_{p, q, r}$. After some algebraic simplifications, we will get the M-polynomial of first Zagreb index for $S_{p, q, r}$ as follows:

$$
P_{M_{1}}\left(S_{p, q, r}\right)=192 p q r x^{4} y^{4}+8(p q+p r+q r)\left(6 x^{3} y^{3}+7 x^{3} y^{4}-12 x^{4} y^{4}\right)+8(p+q+r)\left(3 x^{3} y^{3}-7 x^{3} y^{4}+4 x^{4} y^{4}\right)
$$

Similarly, by using the values of differential operators that are defined in Lemma 1 for $S_{p, q, r}$ in $P_{M_{2}}\left(S_{p, q, r}\right)$
$=\left(D_{x} D_{y}\right)\left(M\left(S_{p, q, r} ; x, y\right)\right)$ and after simplification, we will get the M-polynomial of second Zagreb index for $S_{p, q, r}$ :

$$
P_{M_{2}}\left(S_{p, q, r}\right)=384 p q r x^{4} y^{4}+24(p q+p r+q r)\left(3 x^{3} y^{3}+4 x^{3} y^{4}-8 x^{4} y^{4}\right)+4(p+q+r)\left(9 x^{3} y^{3}-24 x^{3} y^{4}+16 x^{4} y^{4}\right) \text {. }
$$

Theorem 3. Let $S_{p, q, r}$ be a sodalite material, with $p, q, r \geq 1$, and $P_{m_{M}}$ be the M-polynomial of second modified Zagreb index. Then, $P_{m_{M}}\left(S_{p, q, r}\right)$ is

$$
\begin{aligned}
P_{m_{M} M_{2}}\left(S_{p, q, r}\right)= & \frac{3}{2} p q r x^{4} y^{4}+4(p q+p r+q r)\left(\frac{2}{9} x^{3} y^{3}+\frac{1}{8} x^{3} y^{4}-\frac{3}{16} x^{4} y^{4}\right) \\
& +4(p+q+r)\left(\frac{1}{9} x^{3} y^{3}-\frac{1}{8} x^{3} y^{4}+\frac{1}{16} x^{4} y^{4}\right)
\end{aligned}
$$

Proof. According to Definition 3, the formula for the M-polynomial of second modified Zagreb index for $S_{p, q, r}$ is defined as $P_{M_{M}}\left(S_{p, q, r}\right)=\left(S_{x} S_{y}\right)\left(M\left(S_{p, q, r} ; x, y\right)\right)$, by using the integral operators that are defined in Lemma 2 for $S_{p, q, r}$. After some algebraic simplifications, we will get the M-polynomial of second modified Zagreb index for $S_{p, q, r}$ as 


$$
P_{m_{M}}\left(S_{p, q, r}\right)=\frac{3}{2} p q r x^{4} y^{4}+4(p q+p r+q r)\left(\frac{2}{9} x^{3} y^{3}+\frac{1}{8} x^{3} y^{4}-\frac{3}{16} x^{4} y^{4}\right)+4(p+q+r)\left(\frac{1}{9} x^{3} y^{3}-\frac{1}{8} x^{3} y^{4}+\frac{1}{16} x^{4} y^{4}\right)
$$

Theorem 4. Let $S_{p, q, r}$ be a sodalite material, with $p, q, r \geq 1$, and $P_{R_{\alpha}}$ be the M-polynomial of general Randic index. Then, $P_{R_{\alpha}}\left(S_{p, q, r}\right)$ is

$$
\begin{aligned}
P_{R_{\alpha}}\left(S_{p, q, r}\right)= & 16^{\alpha} \times 24 p q r x^{4} y^{4}+4(p q+p r+q r)\left(9^{\alpha} \times 2 x^{3} y^{3}+12^{\alpha} \times 2 x^{3} y^{4}-16^{\alpha} \times 3 x^{4} y^{4}\right) \\
& +4(p q+p r+q r)\left(9^{\alpha} x^{3} y^{3}-12^{\alpha} \times 2 x^{3} y^{4}+16^{\alpha} x^{4} y^{4}\right) .
\end{aligned}
$$

Proof. According to Definition 4, the formula for the M-polynomial of general Randić index for $S_{p, q, r}$ is defined as $P_{R_{\alpha}}\left(S_{p, q, r}\right)=\left(D_{x}^{\alpha} D_{y}^{\alpha}\right)\left(M\left(S_{p, q, r} ; x, y\right)\right)$, by using the differential operators that are defined in Lemma 1 for $S_{p, q, r}$. After some algebraic simplifications, we will get the M-polynomial of general Randić index for $S_{p, q, r}$ as

$$
\begin{aligned}
P_{R_{\alpha}}\left(S_{p, q, r}\right)= & 16^{\alpha} \times 24 p q r x^{4} y^{4}+4(p q+p r+q r)\left(9^{\alpha} \times 2 x^{3} y^{3}+12^{\alpha} \times 2 x^{3} y^{4}-16^{\alpha} \times 3 x^{4} y^{4}\right) \\
& +4(p+q+r)\left(9^{\alpha} x^{3} y^{3}-12^{\alpha} \times 2 x^{3} y^{4}+16^{\alpha} x^{4} y^{4}\right) .
\end{aligned}
$$

Theorem 5. Let $S_{p, q, r}$ be a sodalite material, with $p, q, r \geq 1$, and $P_{I R_{\alpha}}$ be the M-polynomial of general inverse Randic index. Then, $P_{I R_{\alpha}}\left(S_{p, q, r}\right)$ is

$$
\begin{aligned}
P_{\mathrm{IR}_{\alpha}}\left(S_{p, q, r}\right)= & \frac{24}{16^{\alpha}} p q r x^{4} y^{4}+4(p q+p r+q r)\left(\frac{2}{9^{\alpha}} x^{3} y^{3}+\frac{2}{12^{\alpha}} x^{3} y^{4}-\frac{3}{16^{\alpha}} x^{4} y^{4}\right) \\
& +4(p+q+r)\left(\frac{1}{9^{\alpha}} x^{3} y^{3}-\frac{2}{12^{\alpha}} x^{3} y^{4}+\frac{1}{16^{\alpha}} x^{4} y^{4}\right) .
\end{aligned}
$$

Proof. According to Definition 4, the formula for the M-polynomial of general inverse Randić index for $S_{p, q, r}$ is defined as $P_{\mathrm{IR}_{\alpha}}\left(S_{p, q, r}\right)=\left(S_{x}^{\alpha} S_{y}^{\alpha}\right)\left(M\left(S_{p, q, r} ; x, y\right)\right)$, by using the integral operators that are defined in Lemma 2 for $S_{p, q, r}$. After some algebraic simplifications, we will get the M-polynomial of general inverse Randić index for $S_{p, q, r}$ as

$P_{\mathrm{IR}_{\alpha}}\left(S_{p, q, r}\right)=\frac{24}{16^{\alpha}} p q r x^{4} y^{4}+4(p q+p r+q r)\left(\frac{2}{9^{\alpha}} x^{3} y^{3}+\frac{2}{12^{\alpha}} x^{3} y^{4}-\frac{3}{16^{\alpha}} x^{4} y^{4}\right)+4(p+q+r)\left(\frac{1}{9^{\alpha}} x^{3} y^{3}-\frac{2}{12^{\alpha}} x^{3} y^{4}+\frac{1}{16^{\alpha}} x^{4} y^{4}\right)$

\section{Results of Topological Indices from M-Polynomials on Sodalite Materials}

Lemma 3. Let $S_{p, q, r}$ be a sodalite material, with $p, q, r \geq 1$. Then,

$$
\begin{aligned}
& {\left[D_{x}\right]_{(x, y)=(1,1)}=96 p q r+4(p+q+r)} \\
& {\left[D_{y}\right]_{(x, y)=(1,1)}=96 p q r+8(p q+p r+q r)-4(p+q+r) .}
\end{aligned}
$$

Proof. Differentiating equation (8) with respect to $x$ and multiplying the result with $x$, we get $D_{x}$. Similarly, differentiating equation (8) with respect to $y$ and multiplying the result with $y$, we get $D_{y}$. After that in both equations, substituting $(x, y)=(1,1)$ and simplifying, we can easily obtain $\left[D_{x}\right]_{(x, y)=(1,1)}$ and $\left[D_{y}\right]_{(x, y)=(1,1)}$.

Lemma 4. Let $S_{p, q, r}$ be a sodalite material, with $p, q, r \geq 1$. Then, 


$$
\begin{aligned}
& {\left[S_{x}\right]_{(x, y)=(1,1)}=6 p q r+\frac{7}{3}(p q+p r+q r)-\frac{1}{3}(p+q+r),} \\
& {\left[S_{y}\right]_{(x, y)=(1,1)}=6 p q r+\frac{5}{3}(p q+p r+q r)+\frac{1}{3}(p+q+r) .}
\end{aligned}
$$

Proof. By putting the value of general M-polynomial which is defined in equation (8) in equation (6), after simplification, we get $S_{x}$. Similarly, by putting the value of general M-polynomial in equation (7), after simplification, we get $S_{y}$. After simplification, we obtain $S_{y}$. After that, in both equations, substituting $(x, y)=(1,1)$ and simplifying, we can easily obtain $\left[S_{x}\right]_{(x, y)=(1,1)}$ and $\left[S_{y}\right]_{(x, y)=(1,1)}$.

Now, we will use these absolute values from the differential and integral operators which are from Lemmas 3 and 4 to obtain our main results. In the following theorem, we determined the topological indices from M-polynomial of first and second Zagreb indices.

Theorem 6. Let $S_{p, q, r}$ be a sodalite material, with $p, q, r \geq 1$, and $M_{1}, M_{2}$ be the first and second Zagreb indices. Then, $M_{1}\left(S_{p, q, r}\right)$ and $M_{2}\left(S_{p, q, r}\right)$ are

$$
\begin{aligned}
& M_{1}\left(S_{p, q, r}\right)=192 p q r+8(p q+p r+q r) \\
& M_{2}\left(S_{p, q, r}\right)=384 p q r-24(p q+p r+q r)+4(p+q+r) .
\end{aligned}
$$

Proof. According to Definition 2, the formula for the M-polynomial of first Zagreb index for $S_{p, q, r}$ is defined as $P_{M_{1}}\left(S_{p, q, r}\right)=\left(D_{x}+D_{y}\right)\left(M\left(S_{p, q, r} ; x, y\right)\right)$, by using the differential operators that are defined in Lemma 1 for $S_{p, q, r}$. After some algebraic simplifications, we will get the M-polynomial of first Zagreb index for $S_{p, q, r}$.

Now using Lemma 3 and evaluating it in Definition 2 and after some algebraic simplifications, we will get the first Zagreb index for $S_{p, q, r}$ as

$$
M_{1}\left(S_{p, q, r}\right)=192 p q r+8(p q+p r+q r)
$$

Similarly, by using the absolute values of differential operators that are defined in Lemma 3 for $S_{p, q, r}$ in $P_{M_{2}}\left(S_{p, q, r}\right)=\left(D_{x} D_{y}\right)\left(M\left(S_{p, q, r} ; x, y\right)\right)$ and after simplification, we will get the second Zagreb index for $S_{p, q, r}$ :

$$
M_{2}\left(S_{p, q, r}\right)=384 p q r-24(p q+p r+q r)+4(p+q+r) .
$$

Theorem 7. Let $S_{p, q, r}$ be a sodalite material, with $p, q, r \geq 1$, and ${ }^{m} M_{2}$ be the second modified Zagreb index. Then, ${ }^{m} M_{2}\left(S_{p, q, r}\right)$ is

$$
{ }^{m} M_{2}\left(S_{p, q, r}\right)=\frac{3}{2} p q r+\frac{23}{36}(p q+p r+q r)+\frac{7}{36}(p+q+r) .
$$

Proof. According to Definition 3, the formula for the M-polynomial of second modified Zagreb index for $S_{p, q, r}$ is defined as ${ }_{m_{2}}\left(S_{p, q, r}\right)=\left(S_{x} S_{y}\right)\left(M\left(S_{p, q, r} ; x, y\right)\right)$, by using the integral operators that are defined in Lemma 2 for $S_{p, q, r}$. After some algebraic simplifications, we will get the M-polynomial of second modified Zagreb index for $S_{p, q, r}$.

Now using Lemma 4 and evaluating it in Definition 3 and after some algebraic simplifications, we will get the modified Zagreb index for $S_{p, q, r}$ as

$$
\left\{{ }^{m} M_{1}\right\}\left(S_{p, q, r}\right)=\frac{3}{2} p q r+\frac{23}{36}(p q+p r+q r)+\frac{7}{36}(p+q+r) .
$$

Theorem 8. Let $S_{p, q, r}$ be a sodalite material, with $p, q, r \geq 1$, and $R_{\alpha}$ be the general Randic index; then, $R_{\alpha}\left(S_{p, q, r}\right)$ is

$$
\begin{aligned}
R_{\alpha}\left(S_{p, q, r}\right)= & 16^{\alpha} \times 24 p q r+4(p q+p r+q r) \\
& \left(9^{\alpha} \times 2+12^{\alpha} \times 2-16^{\alpha} \times 3\right) \\
& +4(p q+p r+q r)\left(9^{\alpha}-12^{\alpha} \times 2+16^{\alpha}\right) .
\end{aligned}
$$

Proof. According to Definition 4, the formula for the M-polynomial of general Randić index for $S_{p, q, r}$ is defined as $R_{\alpha}\left(S_{p, q, r}\right)=\left(D_{x}^{\alpha} D_{y}^{\alpha}\right)\left(M\left(S_{p, q, r} ; x, y\right)\right)$, by using the differential operators that are defined in Lemma 1 for $S_{p, q, r}$. After some algebraic simplifications, we will get the M-polynomial of general Randić index for $S_{p, q, r}$.

Now using Lemma 3 and evaluating it in Definition 4 and after some algebraic simplifications, we will get the general Randić index for $S_{p, q, r}$ as

$$
\begin{aligned}
R_{\alpha}\left(S_{p, q, r}\right)= & 16^{\alpha} \times 24 p q r+4(p q+p r+q r) \\
& \cdot\left(9^{\alpha} \times 2+12^{\alpha} \times 2-16^{\alpha} \times 3\right) \\
& +4(p q+p r+q r)\left(9^{\alpha}-12^{\alpha} \times 2+16^{\alpha}\right) .
\end{aligned}
$$

Theorem 9. Let $S_{p, q, r}$ be a sodalite material, with $p, q, r \geq 1$, and $R_{\alpha}$ be the general inverse Randic index; then, $\operatorname{IR}_{\alpha}\left(S_{p, q, r}\right)$ is

$$
\begin{aligned}
\operatorname{IR}_{\alpha}\left(S_{p, q, r}\right)= & \frac{24}{16^{\alpha}} p q r+4(p q+p r+q r)\left(\frac{2}{9^{\alpha}}+\frac{2}{12^{\alpha}}-\frac{3}{16^{\alpha}}\right) \\
& +4(p+q+r)\left(\frac{1}{9^{\alpha}}-\frac{2}{12^{\alpha}}+\frac{1}{16^{\alpha}}\right) .
\end{aligned}
$$

Proof. According to Definition 4, the formula for the M-polynomial of general inverse Randić index for $S_{p, q, r}$ is defined as $\operatorname{IR}_{\alpha}\left(S_{p, q, r}\right)=\left(S_{x}^{\alpha} S_{y}^{\alpha}\right)\left(M\left(S_{p, q, r} ; x, y\right)\right)$, by using the 


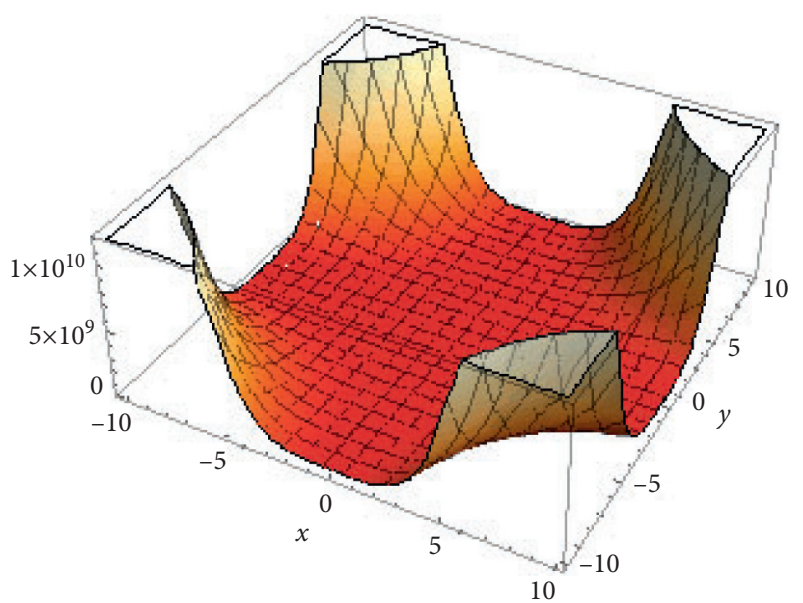

Figure 2: General M-polynomial for three-dimensional sodalite network $S_{4,4,4}$.

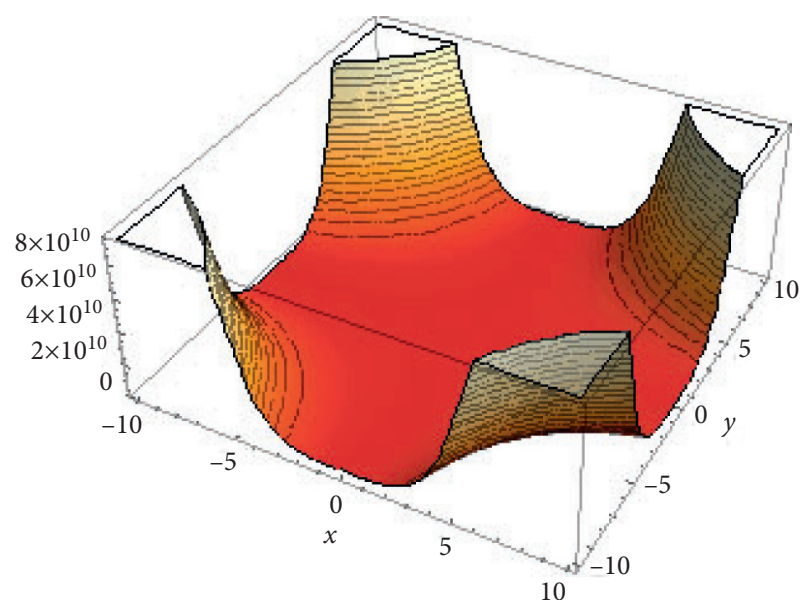

Figure 3: First Zagreb M-polynomial for three-dimensional sodalite network $S_{4,4,4}$.

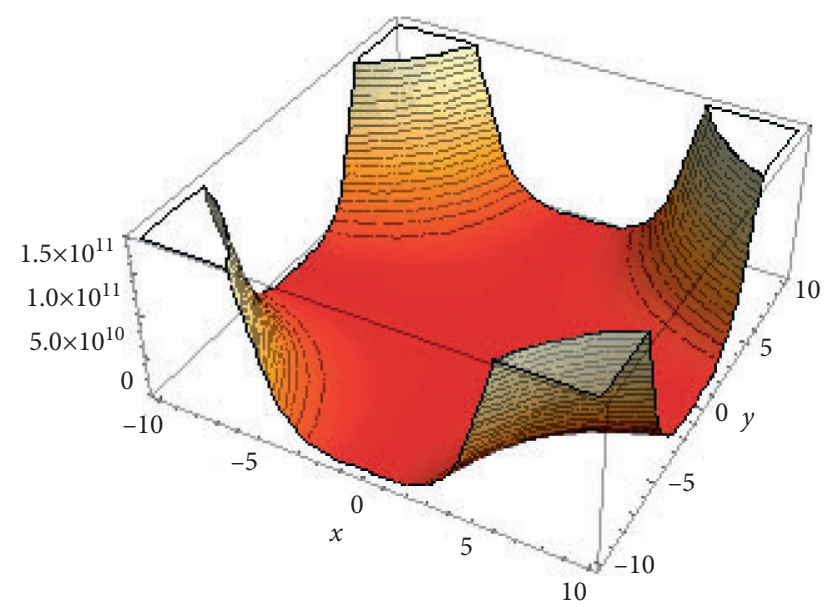

FIgURE 4: Second Zagreb M-polynomial for three-dimensional sodalite network $S_{4,4,4}$.

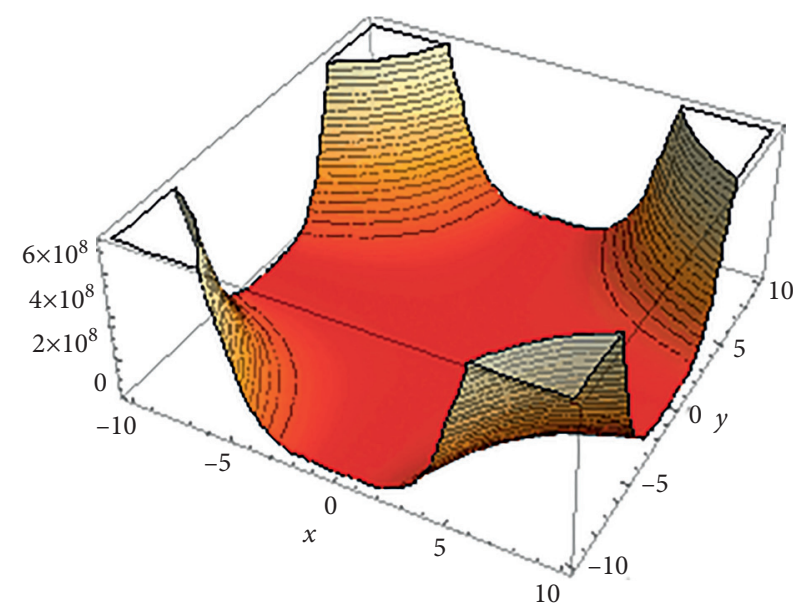

FIgUre 5: Modified Zagreb M-polynomial for three-dimensional sodalite network $S_{4,4,4}$.

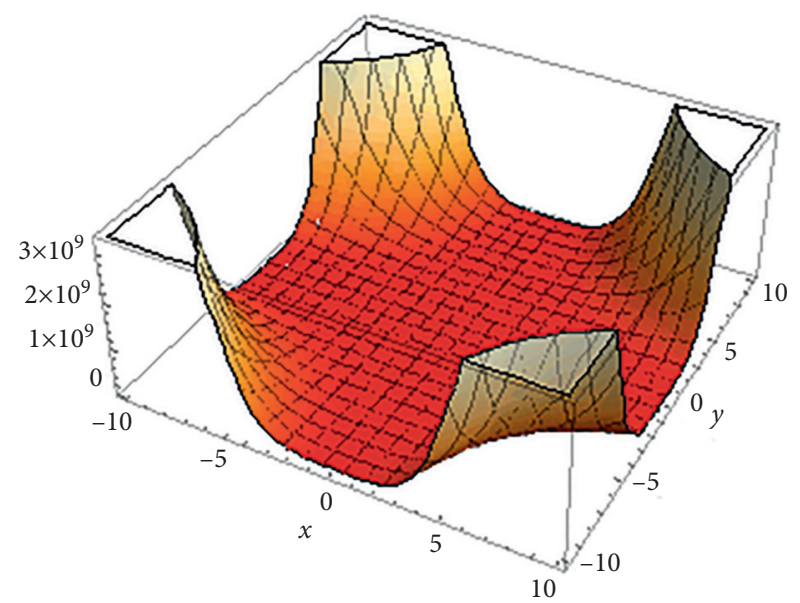

Figure 6: General Randić M-polynomial for three-dimensional sodalite network $S_{4,4,4}$ and $\alpha=-(1 / 2)$.

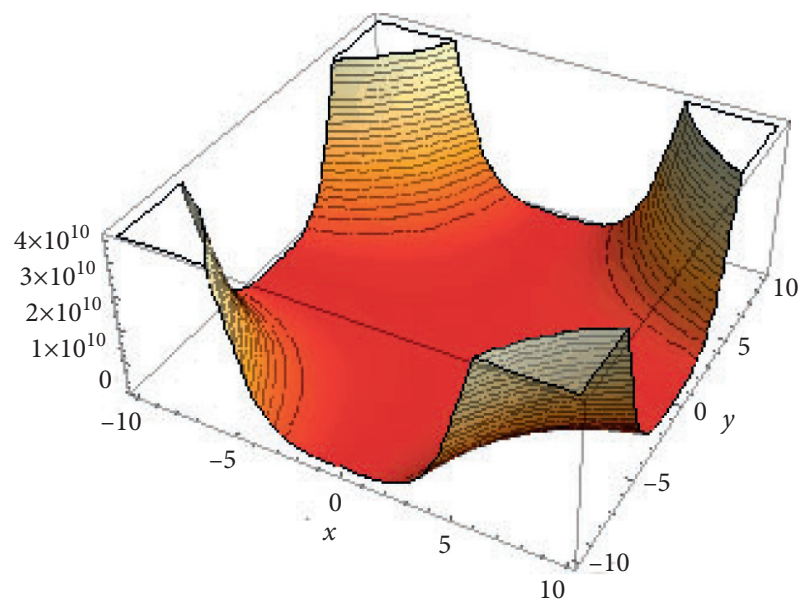

Figure 7: General inverse Randić M-polynomial for three-dimensional sodalite network $S_{4,4,4}$ and $\alpha=-(1 / 2)$. 


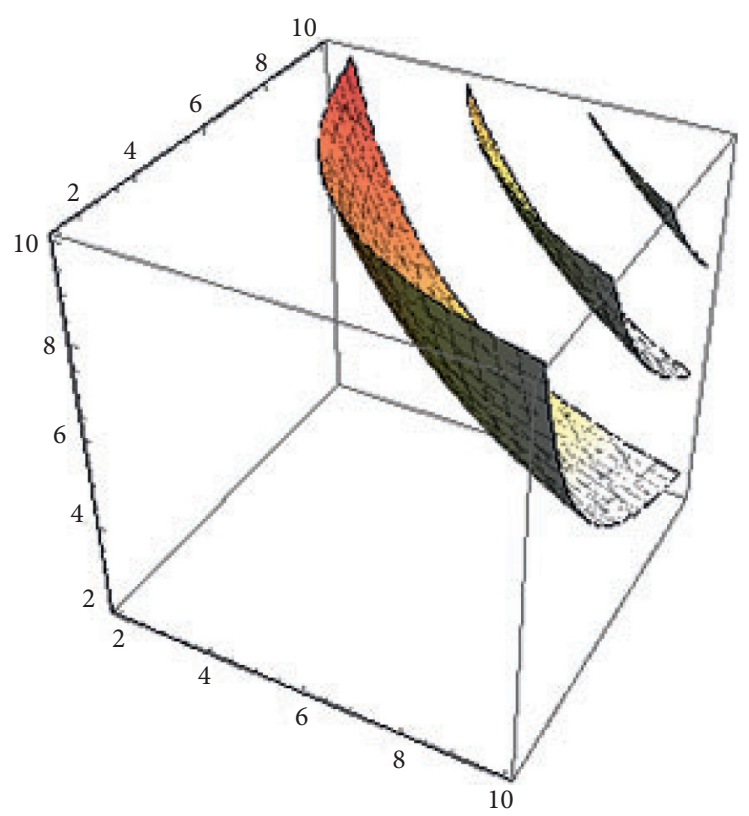

FIGURE 8: Contour plot of topological indices for three-dimensional sodalite network $S_{p, q, r}$ and $\alpha=-(1 / 2)$.

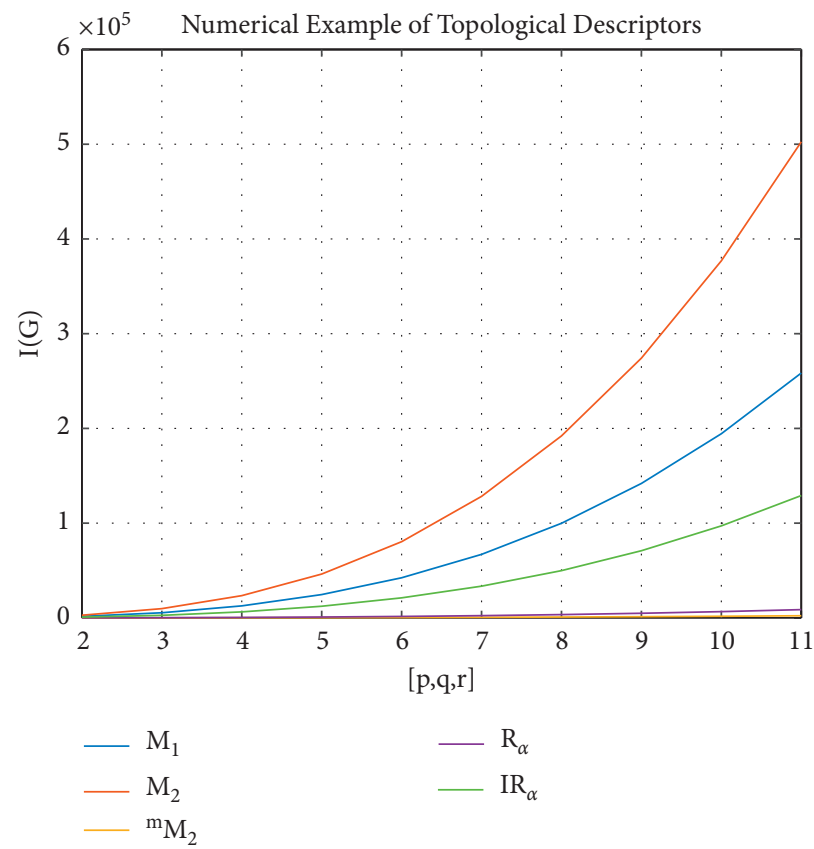

FIgURE 9: Topological indices for three-dimensional sodalite network $S_{p, q, r}$ and $\alpha=-(1 / 2)$. 
integral operators that are defined in Lemma 2 for $S_{p, q, r}$. After some algebraic simplifications, we will get the M-polynomial of general inverse Randić index for $S_{p, q, r}$.

Now using Lemma 4 and evaluating it in Definition 4 and after some algebraic simplifications, we will get the general inverse Randić index for $S_{p, q, r}$ as

$$
\begin{aligned}
\operatorname{IR}_{\alpha}\left(S_{p, q, r}\right)= & \frac{24}{16^{\alpha}} p q r+4(p q+p r+q r)\left(\frac{2}{9^{\alpha}}+\frac{2}{12^{\alpha}}-\frac{3}{16^{\alpha}}\right) \\
& +4(p+q+r)\left(\frac{1}{9^{\alpha}}-\frac{2}{12^{\alpha}}+\frac{1}{16^{\alpha}}\right)
\end{aligned}
$$

\section{Conclusion and Discussion}

In this study, we discussed the most important structure from zeolite structures which is known as sodalite material network and we symbolized the fetched graph of this network by $S_{p, q, r}$, defined in Figure 1 . The moving parameters for this structure are $p, q, r \geq 1$. We study this structure for all these parameters in terms of different $\mathrm{M}$-polynomials and topological indices derived from resulting $\mathrm{M}$-polynomials. Figures 2-7 show the three-dimensional plot of resulting M-polynomials of first, second, and modified Zagreb M-polynomials and general and general inverse Randić M-polynomials, respectively. Figure 8 shows the contour plot of topological index derived from M-polynomials while Figure 9 shows the plot of all the topological indices derived from M-polynomials.

\section{Data Availability}

No data were used to support this study.

\section{Conflicts of Interest}

The authors declare that they have no conflicts of interest.

\section{References}

[1] S. Neeraj, M. L. Noy, C. N. R. Rao, and A. K. Cheetham, "Sodalite networks formed by metal squarates," Solid State Sciences, vol. 4, no. 10, pp. 1231-1236, 2002.

[2] O. Karagiaridi, M. B. Lalonde, W. Bury, A. A. Sarjeant, O. K. Farha, and J. T. Hupp, "Opening ZIF-8: a catalytically active zeolitic imidazolate framework of sodalite topology with unsubstituted linkers," Journal of the American Chemical Society, vol. 134, no. 45, pp. 18790-618796, 2012.

[3] M. Eddaoudi, J. Kim, D. Vodak et al., "Geometric requirements and examples of important structures in the assembly of square building blocks," Proceedings of the National Academy of Sciences, vol. 99, no. 8, pp. 4900-4904, 2002.

[4] C. R. A. Catlow, Modeling of Structure and Reactivity in Zeolites, Academic Press, London, UK, 1992.

[5] A. Dyer, An Introduction to Zeolite Molecular Sieves, WileyInterscience, Chichester, UK, 1988.

[6] F. M. Jaeger, "On the constitution and the structure of ultramarine," Transactions of the Faraday Society, vol. 25, p. 320, 1929.
[7] L. Pauling, "Xxii. The structure of sodalite and helvite," Zeitschrift für Kristallographie - Crystalline Materials, vol. 74, no. 1-6, pp. 213-225, 1930.

[8] S. Prabhu, G. Murugan, M. Cary, M. Arulperumjothi, and J.-B. Liu, "On certain distance and degree based topological indices of zeolite lta frameworks," Materials Research Express, vol. 7, no. 5, Article ID 055006, 2020.

[9] F. Torrens and G. Castellano, "Fractal dimension of active-site models of zeolite catalysts," Journal of Nanomaterials, vol. 2006, Article ID 17052, 9 pages, 2006.

[10] F. Torrens and G. Castellano, "Polarizability characterization of zeolitic br awnsted acidic sites," Recent Progress in Computational Sciences and Engineering, vol. 2, pp. 555-556, 2019.

[11] T. Barth, "The structures of the minerals of the sodalite family," Zeitschrift für Kristallographie - Crystalline Materials, vol. 83, pp. 1-6, 1932.

[12] I. Hassan and H. D. Grundy, "The crystal structures of sodalite-group minerals," Acta Crystallographica Section B Structural Science, vol. 40, no. 1, pp. 6-13, 1984.

[13] D. Taylor, "The sodalite group of minerals," Contributions to Mineralogy and Petrology, vol. 16, no. 2, pp. 172-188, 1967.

[14] M. Arockiaraj, J. Clement, D. Paul, and K. Balasubramanian, "Quantitative structural descriptors of sodalite materials," Journal of Molecular Structure, vol. 1223, Article ID 128766, 2021.

[15] C. Jiri, V. B. Herman, C. Avelino, and S. Ferdi, "Introduction to zeolite science andpractice," Studies in Surface Science and Catalysis, vol. 2, no. 1, pp. 1058-1065, 2007.

[16] M. O’Keeffe, "N-dimensional diamond, sodalite and rare sphere packings," Acta Crystallographica Section A Foundations of Crystallography, vol. 47, no. 6, pp. 748-753, 1991.

[17] M. Irfan, H. U. Rehman, H. Almusawa, S. Rasheed, and I. A. Baloch, "M-polynomials and topological indices for line graphs of chain silicate network and h-naphtalenic nanotubes," Journal of Mathematics, vol. 2021, Article ID 5551825, 11 pages, 2021.

[18] Z. Raza and M. E. K. Sukaiti, "M-polynomial and degree based topological indices of some nanostructures," Symmetry, vol. 12 , no. 5 , p. 831, 2020.

[19] J.-B. Liu, M. Younas, M. Habib, M. Yousaf, and W. Nazeer, "M-polynomials and degree-based topological indices of VC5c7[p, q] and HC5c7[p, q] nanotubes," IEEE Access, vol. 7, pp. 41125-141132, 2019.

[20] M. Cancan, M. Hussain, and H. Ahmad, "Distance and eccentricity based polynomials and indices of $\mathrm{m}$-level Wheel graph," Proyecciones (Antofagasta), vol. 39, no. 4, pp. 869-885, 2020.

[21] M. N. Jahangeer Baig, C. Y. Jung, N. Ahmad, and S. M. Kang, "On the m-polynomials and degree-based topological indices of an important class of graphs," Journal of Discrete Mathematical Sciences and Cryptography, vol. 22, no. 7, pp. 12811288, 2019.

[22] A. Ahmad and S. C. López, "Distance-based topological polynomials associated with zero-divisor graphs," Mathematical Problems in Engineering, vol. 2021, Article ID 4959559, 8 pages, 2021.

[23] M. Riaz, W. Gao, and A. Q. Baig, "M-polynomials and degreebased topological indices of some families of convex polytopes," Open Journal of Mathematical Sciences, vol. 2, no. 1, pp. 18-28, 2018.

[24] C.-P. Li, C. Zhonglin, M. Munir, K. Yasmin, and J. Bao Liu, "M-polynomials and topological indices of linear chains of benzene, napthalene and anthracene," Mathematical Biosciences and Engineering, vol. 17, no. 3, pp. 2384-2398, 2020. 
[25] J. Chidambaraswamy and R. Sitaramachandrarao, "On the probability that the values of $\mathrm{m}$ polynomials have a given g.c.d," Journal of Number Theory, vol. 26, no. 3, pp. 237-245, 1987.

[26] A. Ahmad, M. A. Asim, and M. F. Nadeem, "Polynomials of degree-based indices of metal-organic networks," Combinatorial Chemistry \& High Throughput Screening, vol. 23, 2020.

[27] W. Zhao, M. F. Nadeem, M. Cancan et al., "On degree based topological indices of transition metal-tetra cyano polycyclic benzene organic network," Polycyclic Aromatic Compounds, vol. 2021, Article ID 1900302, 27 pages, 2021.

[28] A. Ahmad, R. Hasni, K. Elahi, and M. A. Asim, "Polynomials of degree-based indices for swapped networks modeled by optical transpose interconnection system," IEEE Access, vol. 8, pp. 214293-214299, 2020.

[29] A. N. A. Koam and A. Ahmad, "Polynomials of degree-based indices for three-dimensional mesh network," Computers, Materials \& Continua, vol. 65, no. 2, pp. 1271-1282, 2020.

[30] A. Ahmad, "Computing the schultz polynomials and indices for ladder related graphs," Proyecciones (Antofagasta), vol. 38, no. 5, pp. 1081-1092, 2019.

[31] M. Ajmal, W. Nazeer, M. Munir, S. Kang, and Y. Kwun, "Some algebraic polynomials and topological indices of generalized prism and toroidal polyhex networks," Symmetry, vol. 9, no. 1, p. 5, 2016.

[32] M. Munir, W. Nazeer, A. Nizami, S. Rafique, and S. Kang, "Mpolynomials and topological indices of titania nanotubes," Symmetry, vol. 8, no. 11, p. 117, 2016.

[33] M. Munir, W. Nazeer, S. Rafique, and S. Kang, "M-polynomial and related topological indices of nanostar dendrimers," Symmetry, vol. 8, no. 9, p. 97, 2016.

[34] M. F. Nadeem, M. Azeem, and H. M. A. Siddiqui, "Comparative study of zagreb indices for capped, semi-capped, and uncapped carbon nanotubes," Polycyclic Aromatic Compounds, vol. 2021, Article ID 1890625, 18 pages, 2021.

[35] M. F. Nadeem, M. Azeem, and I. Farman, "Comparative study of topological indices for capped and uncapped carbon nanotubes," Polycyclic Aromatic Compounds, vol. 2021, Article ID 1903952, 18 pages, 2021.

[36] A. Shabbir, M. F. Nadeem, S. Mukhtar, and A. Raza, "On edge version of some degree-based topological indices of HAC5c7 [p, q] and VC5c7[p, q] nanotubes," Polycyclic Aromatic Compounds, vol. 2020, Article ID 1753220, 17 pages, 2020.

[37] M. F. Nadeem, M. Imran, H. M. A. Siddiqui, M. Azeem, A. Khalil, and Y. Ali, "Topological aspects of metal-organic structure with the help of underlying networks," Arabian Journal of Chemistry, vol. 14, no. 6, Article ID 103157, 2021.

[38] G. Hong, Z. Gu, M. Javaid, H. M. Awais, and M. K. Siddiqui, "Degree-based topological invariants of metal-organic networks," IEEE Access, vol. 8, pp. 68288-68300, 2020.

[39] M. F. Nadeem, S. Zafar, and Z. Zahid, "On topological properties of the line graphs of subdivision graphs of certain nanostructures," Applied Mathematics and Computation, vol. 273, pp. 125-130, 2016.

[40] I. Nadeem, H. Shaker, M. Hussain, and A. Naseem, “Topological indices of para-line graphs of v-phenylenic nanostructures," Open Mathematics, vol. 17, no. 1, pp. 260-266, 2019.

[41] Z. Q. Cai, A. Rauf, M. Ishtiaq, and M. K. Siddiqui, "On vedegree and ev-degree based topological properties of silicon carbide $s i_{2} \dashv c_{3} \dashv i i[p, q]$," Polycyclic Aromatic Compounds, vol. 2020, Article ID 1747095, 15 pages, 2020.

[42] M. Ibrahim, N. Zahra, and M. K. Siddiqui, "On ve-degree and ev-degree based topological indices for the series of benzenoid graphs," Polycyclic Aromatic Compounds, vol. 2021, Article ID 1926294, 10 pages, 2021.

[43] S. Hayat, M. A. Malik, and M. Imran, "Computing topological indices of honeycomb derived networks," Romanian Journal of Information Science and Technology, vol. 18, pp. 144-165, 2015.

[44] J.-H. Tang, M. Habib, M. Younas, M. Yousaf, and W. Nazeer, "Multiplicative topological indices of honeycomb derived networks," Open Physics, vol. 17, no. 1, pp. 16-30, 2019.

[45] M. Javaid and J. Cao, "Computing topological indices of probabilistic neural network," Neural Computing \& Applications, vol. 30, no. 12, pp. 3869-3876, 2017.

[46] J.-B. Liu, Z. Raza, and M. Javaid, "Zagreb connection numbers for cellular neural networks," Discrete Dynamics in Nature and Society, vol. 2020, Article ID 8038304, 8 pages, 2020.

[47] H. Hosoya, "On some counting polynomials in chemistry," Discrete Applied Mathematics, vol. 19, no. 1-3, pp. 239-257, 1988.

[48] E. Deutsch and S. Klavzar, "M-polynomial and degree based topological indices," Iranian Journal of Mathematical Chemistry, vol. 6, pp. 93-102, 2015.

[49] I. Gutman and N. Trinajstc, "Graph theory and molecular orbitals, total $\pi$-electron energy of alternant hydrocarbons," Chemical Physics Letters, vol. 17, pp. 535-538, 1972.

[50] I. Gutman and K. C. Das, "The first zagreb index 30 years after," Match Community of Mathematics and Computational Chemistry, vol. 50, pp. 83-92, 2004.

[51] C. K. Das and I. Gutman, "Some properties of the second zagreb index," Match Community of Mathematics and Computational Chemistry, vol. 52, no. 1, pp. 1-3, 2004.

[52] B. Bollobas and P. Erdos, "Graphs of extremal weights," Ars Combinatoria, vol. 50, pp. 225-233, 1998.

[53] D. Amic, D. Beslo, B. Lucic, S. Nikolic, and N. Trinajstic, "The vertex-connectivity index revisited," Journal of Chemical Information and Computer Sciences, vol. 38, pp. 819-822, 1998. 